\title{
CHARACTERIZATION AND PREVALENCE OF CLINDAMYCIN RESISTANCE STAPHYLOCOCCUS AUREUS FROM CLINICAL SAMPLES OF NATIONAL MEDICAL COLLEGE AND TEACHING HOSPITAL, NEPAL
}

\author{
AMRULLAH SHIDIKI, BIJAY RAJ PANDIT, ASHISH VYAS* \\ Department of Microbiology and Biochemistry, School of Bioengineering and Biosciences, Lovely Professional University, Phagwara, \\ Punjab, India. Email: vyas.Ipu@gmail.com
}

Received: 01 February 2019, Revised and Accepted: 19 March 2019

\begin{abstract}
Objective: Clindamycin is the drug of choice for the treatment of severe form of skin, soft tissue, and blood infections caused by resistant Staphylococcus aureus in the form of methicillin-resistant $S$. aureus (MRSA) and erythromycin-resistant $S$. aureus. In this research, we determine the susceptibility pattern of isolated S. aureus strains against antibiotics and the prevalence of resistant $S$. aureus in the form of MRSA, inducible clindamycin-resistant
\end{abstract} S. aureus (inducible macrolide-lincosamide-streptogramin B [iMLSB]) and constitutive clindamycin-resistant S. aureus (cMLSB).

Methods: A total of 310 isolated S. aureus among 2000 different clinical samples were subjected to oxacillin $(1 \mu \mathrm{g})$ as per the Kirby-Bauer disk diffusion method for MRSA. Clindamycin-resistant either in the form of iMLSB or cMLSB was determined through double disk diffusion method or D-test by use erythromycin $(2 \mu \mathrm{g})$ and clindamycin $(15 \mu \mathrm{g})$ as per the CLSI guidelines.

Results: Out of total S. aureus, MRSA and methicillin-sensitive S. aureus (MSSA) were 78.06\% and 20.64\%, respectively. This study showed that iMLSB and cMLSB were $34.19 \%$ and $23.22 \%$. Both iMLSB and cMLSB were found more among MRSA than MSSA (43.80\%, 26.85\% and 40.62\%, 10.93\%), respectively.

Conclusion: This study helps for the characterization of different resistant strains of $S$. aureus along with the determination of the prevalence rate of these mutant forms causing nosocomial infections.

Keywords: Methicillin-resistant Staphylococcus aureus, Methicillin sensitive Staphylococcus aureus, Inducible macrolide-lincosamide-streptogramin B and constitutive macrolide-lincosamide-streptogramin B, Erythromycin, Oxacillin, Clindamycin.

(c) 2019 The Authors. Published by Innovare Academic Sciences Pvt Ltd. This is an open access article under the CC BY license (http://creativecommons. org/licenses/by/4. 0/) DOI: http://dx.doi.org/10.22159/ajpcr.2019.v12i5.32257

\section{INTRODUCTION}

Staphylococcus aureus is recognized as the causative agent of skin, soft tissue, and systemic infection. It frequently associated with the pus formation in different body sites infections. It is also more common nosocomial as well as opportunistic pathogens [1]. It causes different types of diseases such as skin infections, rhinitis, otitis media infection, mastitis, suppurative wounds osteomyelitis, urinary tract infections, and septic arthritis with life-threatening invasion diseases such as pneumonia, septicemia, endocarditis, bacteremia, and toxic shock syndrome [2].

Origin of methicillin-resistant $S$. aureus (MRSA) made serious problems for the treatment and selection of therapeutics available alternatives for staphylococcal infections [3]. Earlier staphylococcal infections have been treated by the use of erythromycin since three-four decades and become resistant against it has been reported since long [4]. The increase of resistant staphylococcal infection with MRSA has led to renewed interest in the use of macrolide-lincosamide-streptogramin B (MLSB) antibiotics for the treatment of such infections [5]. These family MLSB antibiotics with clindamycin are the preferred antibiotic due to its best pharmacokinetics property [6]

Macrolides (erythromycin, clarithromycin, and roxithromycin) resistance may be constitutive and inducible in the presence of a macrolide inducer being the most common erythromycin [7]. It is the best inducer for the synthesis of erythromycin ribosome methylase (erm) enzyme. In inducible clindamycin resistance S. aureus (iMLSB), erm enzyme only produced in the presence of erythromycin whereas in constitutive phenotype (constitutive clindamycin-resistant $S$. aureus
[cMLSB]) the erm enzyme naturally produced without the presence of inducer. When this enzyme produced through erm gene induced confers cross resistant to other members of group including lincosamide and streptogramin B [8].

The double disk diffusion test (D-test) is used to distinguished inducible clindamycin resistance from constitutive clindamycin-resistant S. aureus among erythromycin-resistant isolates to determine therapeutic for clindamycin to be used as a therapeutic option [9].

In this present research, we aimed to determine the prevalence of MLSBi and MLSBc among hospital-based isolates of S. aureus in the form of MRSA and methicillin-sensitive $S$. aureus (MSSA).

\section{METHODS}

This research included 2000 different clinical samples; pus, blood, urine, sputum, throat swab, pleural fluid, synovial fluid, ascitic fluid, ear swab, cerebrospinal fluid, stool, and high vaginal swab and processed in National Medical College and Teaching Hospital, Birgunj, Nepal from February 2017 to December 2017. Among these, samples 310 (15.5\%) $S$. aureus was isolated by growth on blood agar, nutrient agar, and mannitol salt agar. These isolates were confirmed by use catalase and coagulase test as per the standard operative procedure CLSI [10].

MRSA was identified by the oxacillin disk diffusion method. In this method, 0.5 MacFarland standard inoculums of $S$. aureus was inoculated on Muller-Hinton agar (MHA) media through swabbing and left for $1 \mathrm{~h}$. The oxacillin disc $(1 \mu \mathrm{g})$ was now put on inoculated MHA media and incubated at $37^{\circ} \mathrm{C}$ for $24 \mathrm{~h}$. By the measurement of the zone of diameter, 
Table 1: Sample wise isolation and identification of $S$. aureus

\begin{tabular}{lll}
\hline Clinical samples & Number of samples & S. aureus \\
\hline Ascitic fluid & 7 & 1 \\
Ear swab & 4 & 0 \\
Blood & 529 & 155 \\
Cerebrospinal fluid & 65 & 1 \\
Pus & 488 & 146 \\
Pleural fluid & 21 & 2 \\
Synovial fluid & 8 & 1 \\
Sputum & 136 & 1 \\
Stool & 23 & 0 \\
Throat swab & 8 & 1 \\
Urine & 636 & 2 \\
High vaginal swab & 75 & 0 \\
Total & 2000 & 310 \\
\hline
\end{tabular}

S. aureus: Staphylococcus aureus

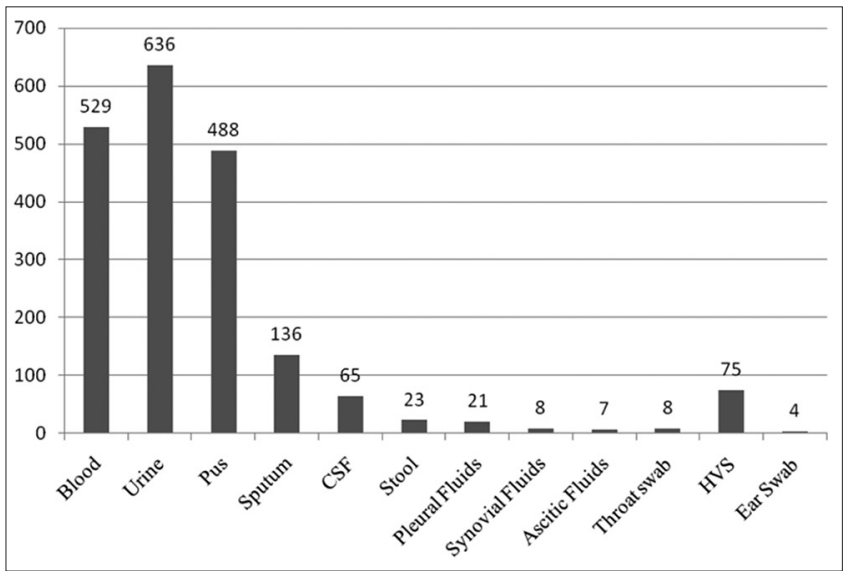

Fig. 1: Distribution of clinical samples. CSF: Cerebrospinal fluid, HVS: High vaginal swab

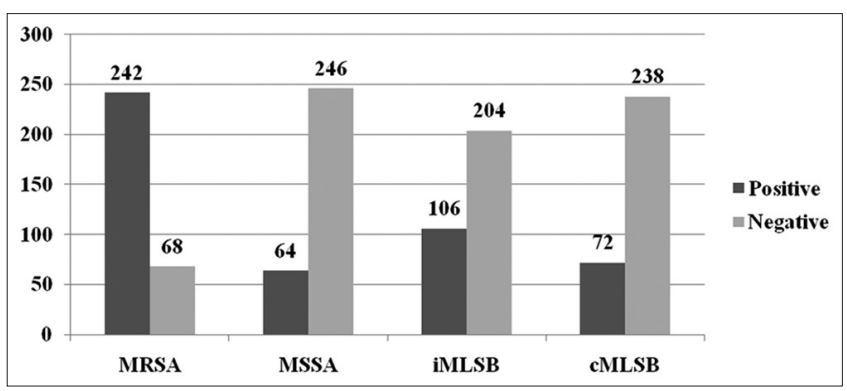

Fig. 2: Selection of resistant strains of Staphylococcus aureus

$\geq 13 \mathrm{~mm}, 11-12 \mathrm{~mm}$, and $\leq 10 \mathrm{~mm}$ around oxacillin disc was considered oxacillin susceptible, intermediate, and resistant, respectively [10].

iMLSB and cMLSB were identified by the use of erythromycin $(15 \mu \mathrm{g})$ and clindamycin $(2 \mu \mathrm{g})$ disks through D-test. In this test 0.5 , MacFarland standard inoculums of $S$. aureus were swabbed on the surface of MHA media. The erythromycin and clindamycin disks were placed at a distance of 15-20 mm distant edge to edge from each other incubated at $37^{\circ} \mathrm{C}$ for $24 \mathrm{~h}$. Through the observation as flattening of the zone of inhibition around the clindamycin proximal to erythromycin disk (D shaped zone of inhibition) indicated as iMLSB phenotypes. There no formation of any zone of inhibition around both antibiotic disks that was defined as cMLSB phenotypes [11].

\section{RESULTS}

Two thousand different clinical samples were processed and the patient samples distribution shown in Fig. 1. Out of total samples, 310 (15.50\%)

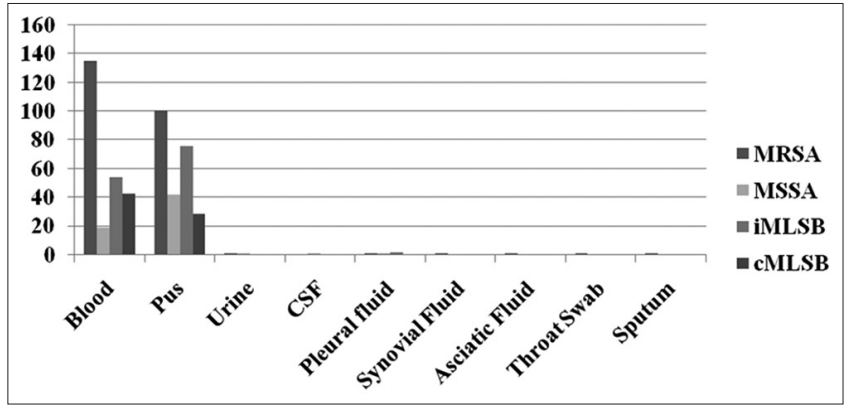

Fig. 3: Distribution of resistant strains of Staphylococcus aureus among clinical samples

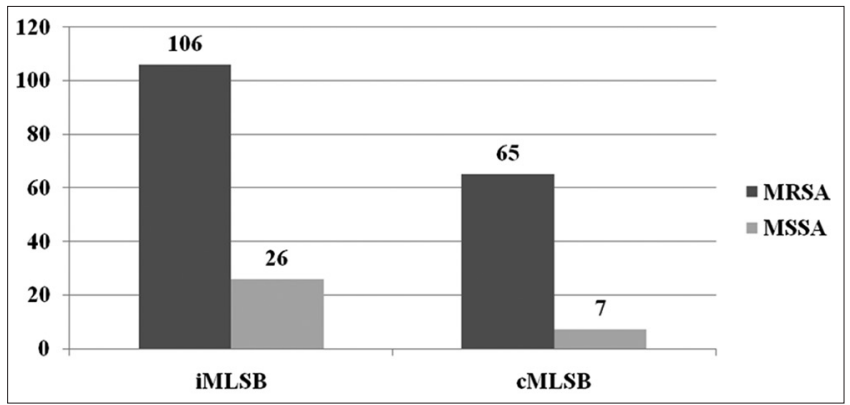

Fig. 4: Selection of inducible macrolide-lincosamidestreptogramin B and constitutive clindamycin-resistant $S$. aureus among methicillin-resistant $S$. aureus and methicillin-sensitive S. aureus

S. aureus were isolated and identified from different samples (Table 1). Among $S$. aureus, MRSA, MSSA, iMLSB, and cMLSB were selected as 242 (78.06\%), 64 (20.64\%), 106 (34.19\%), and 72 (23.22\%), respectively (Fig. 2). MRSA and cMLSB were found more in blood. MSSA and iMLSB were higher in pus than other clinical samples (Fig. 3). Out of total MRSA, 106 (43.80\%) strains were iMLSB and 65 (26.85\%) were cMLSB whereas among MSSA, $26(40.62 \%)$ S. aureus were iMLSB and 7 (10.93\%) were cMLSB (Fig. 4).

\section{DISCUSSION}

Empirical therapy for staphylococcal infection has become more limited due to the emergence and the prevalence of MRSA. Among options available for the treatment of MRSA and MSSA, clindamycin is one of the good alternatives [6]. However, therapeutic failure caused by iMLSB strains is now being reported commonly.

We found a high prevalence of $78.06 \%$ and $20.64 \%$ of MRSA and MSSA among all staphylococcal isolates. A study conducted in Nepal, the prevalence of MRSA was observed as 19\% [12]. Majumder et al. from Assam found 52.9\% MRSA [13]. In our study found $34.19 \%$ iMLSB isolates than $23.22 \%$ cMLSB. A similar study conducted in Turkey observed a prevalence of iMLSB as $21.9 \%$ [14]. Ravisekhar et al. found 21\% iMLSB isolates [15].

It was also observed that the prevalence of iMLSB and CMLSB was higher among MRSA (43.80\% and $26.85 \%$, respectively) as compared to MSSA ( $40.62 \%$ and $10.93 \%)$. This was in concordance with Yilmaz et al. who found $24.4 \%$ iMLSB among MRSA and 14.8\% among MSSA [16]. Rahabar and Hajia reported $22.6 \%$ iMLSB among MRSA and $4 \%$ in MSSA [17].

In one study, all iMLSB shown resistance against macrolides and made either low-level resistance or susceptibility to clindamycin without induction by the use of erythromycin but with induction MICs of clindamycin were noted as an increase from 16 to $256 \mathrm{~g} / \mathrm{ml}$ in iMLSB indicating inducible resistant [18]. Staphylococci making inducible 
resistance to MLS antibiotics are now common to clinical practice, especially in developing countries. There should be cautious for using the clindamycin in a severe case of infections due to making constitutive mutants during the course of clindamycin therapy in patients with iMLSB [19].

At present, some authors suggested less or no use of clindamycin for serious infections with high burden bacteria such as abscess or osteomyelitis [20]. Conversely, all erythromycin-resistant $S$. aureus was labeled as clindamycin resistant would prevent the use of clindamycin in infections caused by clindamycin-susceptible isolates. Clindamycin is now mostly used in non iMLSB or less severe form of infections caused by $S$. aureus.

\section{CONCLUSION}

Although the prevalence rate may differ from hospital to hospital, this study reflects characterization and prevalence of MRSA, MSSA, iMLSB, and cMLSB strains through oxacillin disk diffusion and the D-test at tertiary care hospital. Microbiologists should conform for theses mutants strains among $S$. aureus isolates.

\section{ACKNOWLEDGMENT}

The authors wish to acknowledge to the administrative peoples as well as laboratory workers of the Department of Microbiology of National Medical College and Teaching Hospital, Birgunj, Nepal, for their valuable suggestions and cooperation.

\section{AUTHORS' CONTRIBUTIONS}

Amrullah Shidiki was involved in concepts, design, definition of intellectual content, literature search, clinical studies, experimental studies, data acquisition, data analysis, manuscript preparation, and manuscript editing and was a manuscript review guarantor. Bijay Raj Pandit was supported in concepts, design, definition of intellectual content, literature search, clinical studies, data analysis, manuscript preparation, and manuscript editing. Ashish Vyas was helped in concepts, definition of intellectual content, experimental studies, data analysis, and manuscript editing.

\section{CONFLICTS OF INTEREST}

The authors have not declared any conflicts of interest.

\section{REFERENCES}

1. Tilton F, Kumar RM, Jeyanthi V, Priya LK. In silico interaction analysis of herbal bioactive molecules with penicillin-binding proteins in Staphylococcus aureus. Asian J Pharm Clin Res 2016;9:44-7.

2. DeLeo FR, Chambers HF, Reemergence of antibiotic-resistant Staphylococcus aureus in the genomics era. J Clin Invest 2009;119:2464-74.

3. Bhoosan M, Prasad J, Dutta A, Ke V, Mukhopadhyan C. Reduced susceptibility of MRSA to vancomycin. Int $\mathrm{J}$ Pharm Pharm Sci 2016;8:321-2

4. Patel M, Waites KB, Moser SA, Cloud GA, Hoesley CJ. Prevalence of inducible clindamycin resistance among community and hospitalassociated Staphylococcus aureus isolates. J Clin Microbiol 2006;44:2481-4.

5. Lertcanawanichakul M, Chawawisit K, Choopan A, Nakbud K, Dawveerakul K. Incidence of constitutive and inducible clindamycin resistance in clinical isolates of methicillin resistant Staphylococcus aureus. Walailak J Sci Tech 2007;4:155-63.

6. Fiebelkorn KR, Crawford SA, McElmeel ML, Jorgensen JH. Practical disc diffusion method for detection of inducible clindamycin resistance in Staphylococcus aureus and coagulase negative staphylococci. J Clin Microbiol 2003;41:4740-4

7. Delialioglu N, Aslan G, Ozturk C, Baki V, Sen S. Inducible clindamycin resistance in staphylococci isolated from clinical samples. J Infect Dis 2005;58:104-6.

8. Leclercq R. Mechanisms of resistance to macrolides and lincosamides. Clin Infect Dis 2002;34:482-92.

9. Fiebelkorn KR, Crawford SA, McElmeel ML, Jorgensen JH. Practical disc diffusion method for detection of inducible clindamycin resistance in Staphylococcus aureus and coagulase-negative staphylococci. J Clin Microbiol 2003;41:4740-4

10. CLSI. Performance Standards for Antimicrobial Susceptibility Testing. Wayne, PA: Clinical and Laboratory Standards Institute; 2007.

11. Miller JM, Shah S. Patterns of phenotypic resistance to the macrolide-lincosamide-ketolide-streptogramin group of antibiotics in staphylococci. J Antimicrob Chemother 2000;46:941-9.

12. Bhatt CP, Karki BM, Baral B, Gautam S, Shah A, Chaudhary A. Antibiotic susceptibility pattern of Staphylococcus aureus and methicillin-resistant Staphylococcus aureus in a tertiary care hospital. J. Pathol. Nepal 2014;4:548-551.

13. Majumder D, Bordoloi JN, Phukan AC, Mahanta J. Antimicrobial susceptibility pattern among methicillin resistant Staphylococcus isolates in Assam. IJMM 2001;19:138-140.

14. Gurdal Y, Kemalettin A. Detection and prevalence of inducible clindamycin resistance in staphylococci. J Med Microbiol 2007;56:342-5.

15. Ravisekhar G, Benu D, Srujana M, Arti K. Inducible clindamycin resistance in clinical isolates of Staphylococcus aureus. Indian J Med Res 2006;123:571-3.

16. Yilmaz G, Aydin K, Iskender S, Caylan R, Koksal I. Dete0ction and prevalence of inducible clindamycin resistance in staphylococci. J Med Microbiol 2007;56:342-5.

17. Rahabar M, Hajia M. Inducible clindamycin resistance in Staphylococcus aureus: A cross sectional report. Pak J Biol Sci 2007;10:189-92.

18. Goyal R, Singh NP, Manchanda V, Mathur M. Detection of clindamycin susceptibility in macrolide resistant phenotypes of Staphylococcus aureus. Indian J Med Microbiol, 2004; 22: 251-254.

19. Levin TP, Suh B, Axelrod P, Truant AL, Fekete T. Potential clindamycin resistance in clindamycin susceptible, erythromycin resistant Staphylococcus aureus: Report a clinical failure. Antimicrob Agents Chemother 2005;49:1222-4.

20. Siberry GK, Tekle T, Carroll K, Dick J. Failure of clindamycin treatment of methicillin resistant Staphylococcus aureus expressing inducible clindamycin resistance in vitro. Clin Infect Dis 2003;37:1257-60. 